\title{
Neuro-Fuzzy Network Technique for Semantic Segmentation Robotics
}

\author{
Kanwaljit Kaur \\ Department of Electronics and communication \\ ACET College, Amritsar
}

\author{
Gurjit Singh \\ Department of Electronics and communication \\ ACET College, Amritsar
}

\begin{abstract}
The various techniques are proposed for better semantic segmentation. The neuro-fuzzy technique is proposed for learning common nature between object and structure. The proposed technique work better for robotic environment for fast and efficient results. The proposed technique provides better accuracy as compared to previous technique and work better in semantic segmentation as compared to previous technique.
\end{abstract}

\section{Keywords}

3D entangled, Neuro -Fuzzy Network, segmentation.

\section{INTRODUCTION}

During the last many years, robotics analysis has been geared toward finding solutions to the technical requirements of applied robotics. The evolution of application fields and their sophistication have influenced analysis topics within the robotics community. The direct surrounding of mobile robots is required while making decision in indoor environment since indoor environment are very complex. The robots are required for recognizing the environment for the basic problem of segmentation of observed scène for meaningful semantic parts [2].

The robots are playing important role in image processing technique. The 2-dimensional image computer system is used for human interpretation which are stored in the computer vision community attempts were made to model the human perception process starting with, which is definitively stored in a memory. The various algorithm and technique are used for 3-Dimensional perception to 2-Dimensional of human mind [7].

\section{SEMANTIC SEGMENTATION}

Segmentation is a division of a image into many logical parts but segmentation clearly define the meaning of these parts. On the opposite hand semantic segmentation makes an effort to partition the image into semantically meaning parts also to classify each part into one among pre- determined categories. Semantic segmentation may be a classification task. Semantic segmentation is that the task of cluster components of pictures along that belongs to a similar object category.

While having linguistics segmentation is actually a giant advantage once making an attempt to induce object instances, there are a few of problems: neighbor pixels of a similar category may belong to completely different object instances and regions that aren't connected may Belong to identical object instance. For instance, a tree ahead of a car visually divides the car into two components [8].

For instance, think about a automaton within the indoor scene, so as to securely navigate through the surroundings, the automaton should understand the free area of the scene accurately (geometric structure). Moreover, so as for the automaton to effectively act with the surroundings (e.g., to position a bottle on a table), it should acknowledge the objects within the scene (semantic structure) [9].

\section{3D ENTANGLED FORESTS FOR CLASSIFICATION}

In several recently given approaches within the field of semantic segmentation, RFs are the strategy of alternative for the classification step, sometimes followed by a CRF model to get a sleek final labeling. A regular RF classifier usually processes data points severally from one another, and every information corresponds to a feature vector that is sometimes calculated during a closely finite native region. Therefore, classification results not solely suffer from classification noise; the potential of incorporating a lot of complicated discourse data is additionally terribly restricted. However, the design of the quality RF classifier may be changed to permit for a brand new kind of options known as entangled options, that are ready to make amends for those deficits. particularly, they utilize the learned tree structure and intermediate category distributions, ensuing from coaching the nodes nearer to the foundation node, providing discourse data that helps ripping coaching information once learning deeper levels of the tree. So far, entangled options have solely been utilized in 2-D image house, principally to classify grey scale medical pictures, e.g. from a CT. within the next sections, we tend to take the idea of entanglement to the next level and host 3-D Entangled Forests [2].

\subsection{D entangled features}

A fundamental part of entangled options could be a technique that is ready to be told wherever to search out repeatable and across completely different scenes alternative information points, whose discourse info helps the foremost to predict the present information. As introduced in [10], for 2-D pictures this drawback breaks all the way down to learning 2-D picture element offsets relative to the present picture element to pick out a close-by space to judge the entangled feature on. For 3D purpose clouds, however, the matter gets disproportionately tougher, since information lives in projective area.

\section{RELATED WORK}

[1] Yiyi Liao and Sarath Kodagoda proposed an improvement in Scene classification could be a basic in understanding of environmental task by nowadays. Robotics machine learning language is used for scene understanding with robotics applications the calculated design scene are with better results in semantic segmentation in robotics Daniel Wolf associated Johann Prankl proposed an improvement in novel, fast, and compact technique to enhance semantic segmentation of three-dimensional (3-D) purpose clouds, that is in a position 
to find out and exploit common discourse relations between discovered structures and objects. Introducing three-D Entangled Forests (3-DEF), The idea of entangled options for decision trees to three-D purpose clouds, enabling the classifier not solely to find out, that labels are probably to occur near one another, however also within which specific geometric configuration. Operational on a plane-based illustration of a point cloud, our technique doesn't need a final smoothing step and achieves progressive results on the NYU Depth Dataset in a very single inference step. This compactness successively permits for quick process times, a vital issue to think about for on-line applications on robotic platforms. in a very thorough analysis, The expressiveness of our new three-D entangled feature set and also the importance of spatial context within the scope of semantic segmentation [2]

David Inkyu Kim and Gaurav S. Sukhatme proposed.unique technique for semantic labelling will applied for design changing the result with proposed technique are $81.8 \%$ better than various techniques [3]

Arsalan Mousavian, Jana Koseck and Jyh-Ming Lien Efficient proposed an improvement in drawback of image based mostly \} localization features a long history each in artificial intelligence and pc vision and shares several similarities with image based retrieval drawback. Existing techniques use either native options or (semi)-global image signatures within the context of topological mapping or loop closure detection. Difficulties of the situation recognition drawback are usually stricken by massive look and read point the main task of robotics is semantic central unit. The semantic segmentation work in various fields like as human interaction and automatic working, due to which the accurate results are produced. [5]

Sen Wang and Xinxin Zuo that major work of robotics for indoor location is geometry environment enhancement. And for that surface segmentation is done for RGB-D pictures [6]

\section{PROPOSED METHODOLOGY}

In our proposed methodology minimum and maximum strength of systematic sets are denoted for chosen row and column intensity values for reducing the storage space. For reducing storage space the interim sequence is also stored. On the basis of Intensity value intervals the neuro-fuzzy network procedure are formed for document image for favorable intensity various interval set are formed. The shape and intensity of pixel from various intervals helps in defining the feature of image. The neuro-fuzzy network work on defining various desired intensity layers for retrieving desired results from interval of image portion.

If the pixel with uniform intensity arises they are considered as similar region. The proposed technique neuro-fuzzy network help in identifying this similar region for better selection of region for efficient results.

\subsection{Flow diagram of proposed methodology}

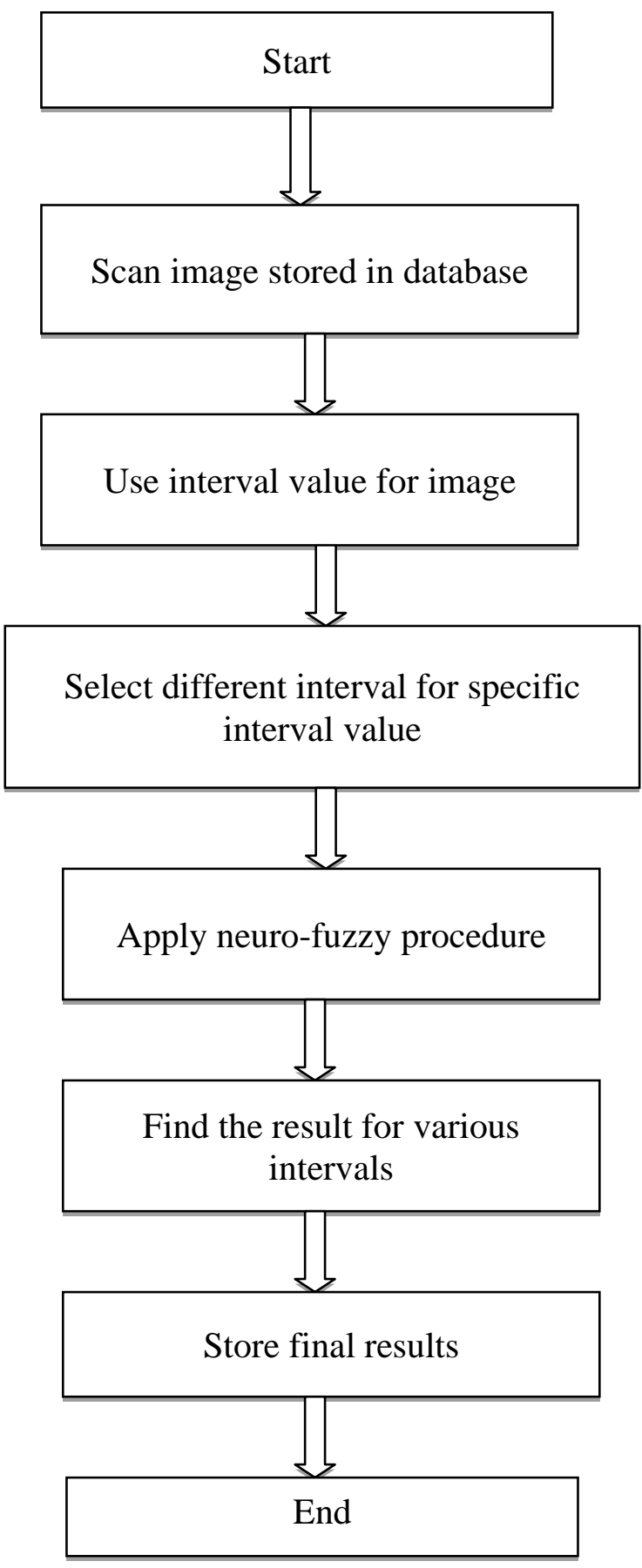

Fig.1 Flow diagram of proposed methodology

\section{RESULTS AND ANALYSIS}

In this section we have discussed our results with the neuro fuzzy technique and also discussed the comparison of both neuro fuzzy and 3D entangled features. 


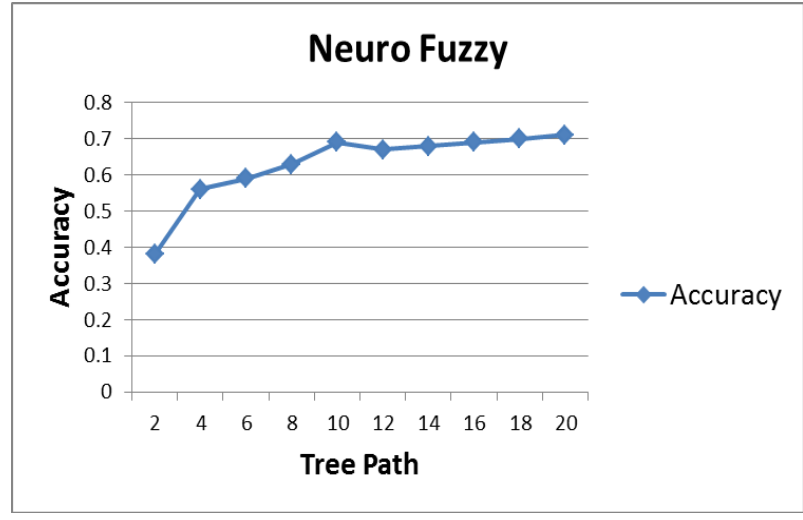

Fig.2 Results of accuracy

Table1. Corresponding results to Tree path

\begin{tabular}{|l|l|}
\hline Tree path & Accuracy \\
\hline 2 & 0.38 \\
\hline 4 & 0.56 \\
\hline 6 & 0.59 \\
\hline 8 & 0.63 \\
\hline 10 & 0.69 \\
\hline 12 & 0.67 \\
\hline 14 & 0.68 \\
\hline 16 & 0.69 \\
\hline 18 & 0.70 \\
\hline 20 & 0.71 \\
\hline
\end{tabular}

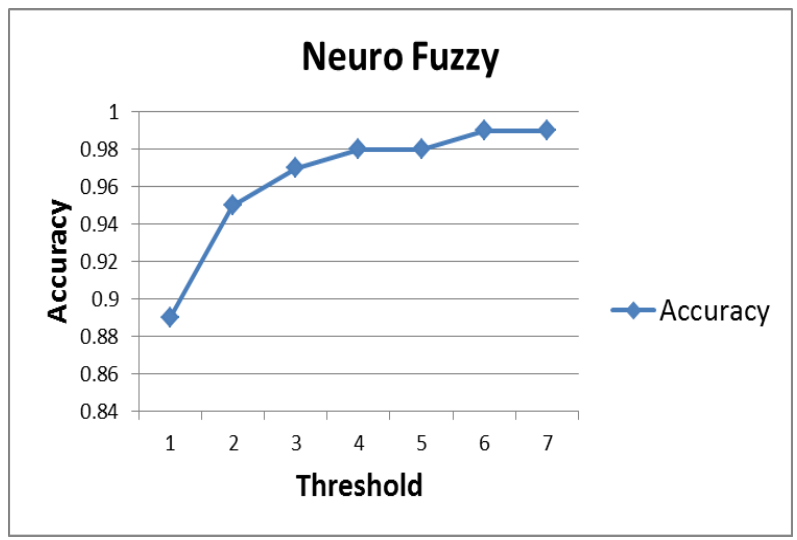

Fig.3 Results with threshold value

Table 2. Corresponding results to Threshold

\begin{tabular}{|l|l|}
\hline Threshold & Accuracy \\
\hline 1 & 0.89 \\
\hline 2 & 0.95 \\
\hline 3 & 0.97 \\
\hline 4 & 0.98 \\
\hline 5 & 0.98 \\
\hline 6 & 0.99 \\
\hline 7 & 0.99 \\
\hline
\end{tabular}

\section{CONCLUSION}

The Neuro-Fuzzy network technique is proposed for efficient semantic segmentation. The proposed technique is capable of finding relation between the objects, structure and indoors. The proposed technique is evaluated on the basis of intervals intensity value for favorable portion for desired results. The proposed technique provides better results as compared to previous technique. In future scope of a work visually convincing result will be used which are more accurate in semantic segmentation. These results will be shown by using optical flow.

\section{REFERENCES}

[1] Yiyi Liao, Sarath Kodagoda, Yue Wang, Lei Shi and Yong Liu, "Understand Scene Categories by Objects: A Semantic Regularized Scene Classifier Using Convolutional Neural Networks", International Conference on Robotics and Automation (ICRA) Stockholm, 2016, pp.2318-2325.

[2] Daniel Wolf, Johann Prank, and Markus Vincze "Enhancing Semantic Segmentation for Robotics: The Power of 3-D Entangled Forests", IEEE ROBOTICS AND AUTOMATION LETTERS, Volume 1, 2016, pp.49-56

[3] David Inkyu Kim and Gaurav S. Sukhatme, "Semantic Labeling of 3D Point Clouds with Object Affordance for Robot Manipulation", IEEE International Conference on Robotics \& Automation (ICRA), 2014, pp.55785584.

[4] Arsalan Mousavian , Jana Koseck and Jyh-Ming LienEfficient "Semantically Guided Location Recognition for Outdoors Scenes", IEEE International Conference on Robotics and Automation (ICRA), 2015, pp.4882-4889.

[5] Richard Bormann and Florian Jordan, Wenzhe Li, Joshua Hampp, and Martin Hagele , "Room Segmentation: Survey, Implementation, and Analysis", 2016, pp.10191026.

[6] Sen Wang, Xinxin Zuo,Weiwei Yu and Runxiao Wang, "Towards robotic semantic segmentation of supporting surfaces", IEEE International Conference on Computational Intelligence \& Communication Technology, 2015, pp.775-779.

[7] Saurabh Gupta, Ross Girshick, Pablo Arbelaez , and Jitendra Malik, "Learning Rich Features from RGB-D Images for Object Detection and Segmentation",2014,pp.1-16.

[8] Hannes Schulz, Benedikt Waldvogel, Rasha Sheikh, and Sven Behnke, "CURFIL: Random Forests for Image Labeling on GPU", International Conference on Computer Vision, 2015.

[9] Mircea Serban Pavel, Hannes Schulz, Sven Behnke, and Sven Behnke, "Recurrent Convolutional Neural Networks for Object-Class Segmentation of RGB-D Video", International Joint Conference on Neural Networks (IJCNN), 2015.

[10] Andreas C. Muller" and Sven Behnke, "Learning DepthSensitive Conditional Random Fields for Semantic Segmentation of RGB-D Images", International Conference on Robotics \& Automation (ICRA), 2014, pp.6232-6237. 